\title{
An analysis of knowledge sharing behaviors in requirement engineering through social media
}

\begin{abstract}
Requirement engineering activities are very important for software development. However, it is difficult to capture and analyze requirements correctly and completely, and also, how to develop a set of accurate requirements is becoming an important issue. Despite there are many methods developed in order to gain more accurate requirements, there is lacking research highlight capturing more comprehensive requirements through knowledge sharing by using social media. Knowledge sharing is an interactive process which involves exchanging relevant knowledge such as information, idea, experience, knowledge know-how, and procedures from broader scope of resources in requirements engineering. Through knowledge sharing, the quality of communications between analysts and users is improved to make a good understanding of the users requirements. So that, the software developers can produce higher qualitative product. This paper proposes a flow of knowledge sharing activities through social media, and analyzes the knowledge sharing behaviors in process of requirements engineering.
\end{abstract}

Keyword: Knowledge sharing; Knowledge sharing behavior; Requirement engineering; Social media 